$$
\text { CONF-980560-- }
$$

\title{
H-MODE PEDESTAL CHARACTERISTICS
SHAPE DISCHARGES ON DIII-D
}

$\begin{array}{rr}\text { by } & \text { SEP } 281998\end{array}$ RECEIVED SEP 281990 A.W. LEONARD, R. MAINGI, R.L. MILLER, G.D. PORTER, G.M. STAEBLER, and A.D. TURNBULL

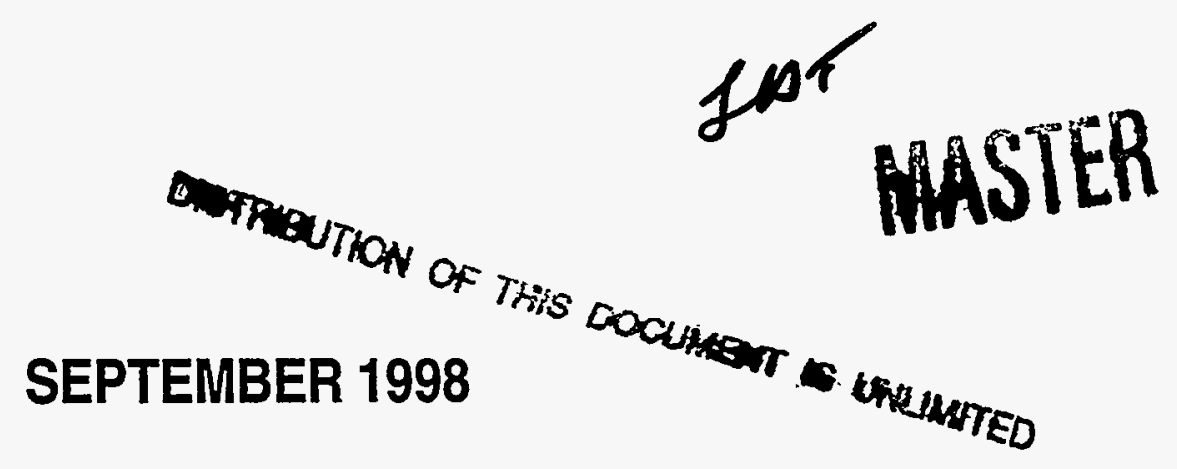




\section{DISCLAIMER}

This report was prepared as an account of work sponsored by an agency of the United States Government. Neither the United States Government nor any agency thereof, nor any of their employees, makes any warranty, express or implied, or assumes any legal liability or responsibility for the accuracy, completeness, or usefulness of any information, apparatus, product, or process disclosed, or represents that its use would not infringe privately owned rights. Reference herein to any specific commercial product, process, or service by trade name, trademark, manufacturer, or otherwise, does not necessarily constitute or imply its endorsement, recommendation, or favoring by the United States Government or any agency thereof. The views and opinions of authors expressed herein do not necessarily state or reflect those of the United States Government or any agency thereof. 


\section{DISCLAIMER}

Portions of this document may be illegible in electronic image products. Images are produced from the best available original document. 


\title{
H-MODE PEDESTAL CHARACTERISTICS IN ITER SHAPE DISCHARGES ON DIII-D
}

by
T.H. OSBORNE, K.H. BURRELL, R.J. GROEBNER, L.L. LAO,
A.W. LEONARD, R. MAINGI, † R.L. MILLER, G.D. PORTER,
G.M. STAEBLER, and A.D. TURNBULL

This is a preprint of a paper to be presented at the 13th International Conference on Plasma Surface Interactions in Controlled Fusion Devices, May 18-23, 1998, San Diego, California and to be published in Journal of Nuclear Materials.

\author{
†Oak Ridge National Laboratory \\ ‡Lawrence Livermore National Laboratory
}

\author{
Work supported by \\ the U.S. Department of Energy \\ under Contracts DE-AC03-89ER51114, DE-AC05-96-OR22464, \\ and W-7405-ENG-48
}

GA PROJECT 3466

SEPTEMBER 1998 


\section{ABSTRACT}

Characteristics of the H-mode pedestal are studied in Type I ELM discharges with ITER cross-sectional shape and aspect ratio. The scaling of the width of the edge steep gradient region, $\delta$, which is most consistent with the data is with the normalized edge pressure, $\left(\beta_{\mathrm{POL}}^{\mathrm{PED}}\right)^{0.4}$. Fits of $\delta$ to a function of temperature, such as $\rho_{\text {PoL }}$, are ruled out in divertor pumping experiments. The edge pressure gradient is found to scale as would be expected from infinite $\mathrm{n}$ ballooning mode theory; however, the value of the pressure gradient exceeds the calculated first stable limit by more than a factor of 2 in some discharges. This high edge pressure gradient is consistent with access to the second stable regime for ideal ballooning for surfaces near the edge. In lower $q$ discharges, including discharges at the ITER value of $q$, edge second stability requires significant edge current density. Transport simulations give edge bootstrap current of sufficient magnitude to open second stable access in these discharges. Ideal kink analysis using current density profiles including edge bootstrap current indicate that before the ELM these discharges may be unstable to low $\mathrm{n}$, edge localized modes. 


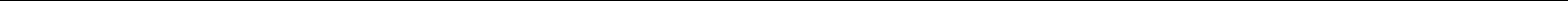




\section{INTRODUCTION}

A defining feature of $\mathrm{H}$-mode is the existence of a transport barrier near the plasma boundary characterized by a pedestal at the top of a steep gradient. The characteristics of the $\mathrm{H}$-mode pedestal may be important in determining the overall plasma performance. Theory based transport models, which apply ot the region inside the $\mathrm{H}$-mode transport barrier, that include nonlinear, electrostatic ion temperature gradient (ITG) mode and trapped electron mode (TEM) physics [1,2], predict a high level of turbulence above a critical temperature gradient scale length. This "stiffness" of the temperature profile implies that the core transport coefficients are reduced strongly with increasing edge pedestal temperature. When applied to ITER [3] these models give an edge pedestal temperature requirement of roughly $4 \mathrm{keV}$ to achieve ITER's design goal of $1500 \mathrm{MW}$; at $1 \mathrm{keV}$ the fusion power output would be reduced dramatically to only about $200 \mathrm{MW}$. Although models based on other instabilities give less [4] or no [5] "stiffness" it is difficult to select the more accurate model based on current experimental results [3]. The $\mathrm{H}$-mode edge characteristics are also important in their connection to ELM stability and energy loss. In DIII-D discharges the energy lost from the plasma core at a Type I ELM, DEELM , is proportional to the energy in the $\mathrm{H}$-mode pedestal [6]. Estimates for ITER are in the range of $\mathrm{DE}_{\mathrm{ELM}} \approx 10 \mathrm{MJ}$ [7] which could result in significant erosion of the divertor plates.

This paper primarily describes experiments in DUI-D employing discharges with ITER cross sectional shape and aspect ratio $\left(R_{D I I-D} / R_{I T E R}=0.2\right)$. In these experiments the plasma current and toroidal field were varied by a factor of two as was the $q, 3<q_{95}<6$, $\mathrm{q}_{95}=\mathrm{q}\left(\frac{\psi-\psi_{\mathrm{AXIS}}}{\psi-\psi_{\text {SEP }}}=0.95\right)$, and $\psi$ is the poloidal flux, qITER $_{3} \cong 3$. The discharges were neutral beam heated with power flux at plasma boundary $0.06<\mathrm{P} / \mathrm{S}\left(\mathrm{MW} / \mathrm{m}^{2}\right)<0.3$, where $(\mathrm{P} / \mathrm{S})_{\mathrm{ITER}} \approx 0.2$ from auxiliary heating. The density was in the range $0.2<\mathrm{n}_{\mathrm{G}}=\mathrm{n} / \mathrm{n}_{\mathrm{GREENWALD}}<0.7$, while $\mathrm{n}_{\mathrm{G}-}$ ITER $\approx 1.0$. These were gas puff fueled discharges with the $\nabla B$ drift toward the $\mathrm{X}$-point. We separated our study of the $\mathrm{H}$-mode pedestal parameters into analysis of the scaling of the width of the steep pressure gradient region, which is expected to be set by turbulence suppression physics [8], and the magnitude of the gradient, which may be limited by the ELM instability [9]. 


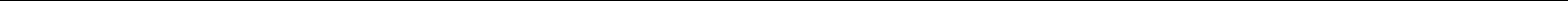




\section{SCALING OF H-MODE TRANSPORT BARRIER WIDTH}

The $\mathrm{H}$-mode transport barrier is thought to be the result of the suppression of turbulence in the plasma edge. The nature of the turbulence and the processes which lead to its suppression are topics of current research, however experiments have shown that a sheared $E_{R} \times B$ flow forms in the edge of sufficient magnitude to suppress the observed fluctuations [8]. The width of the $\mathrm{H}$-mode transport barrier may be set by the innermost point at which turbulence suppression can occur. In this paper we shall examine two theoretical predictions for the barrier width, and also derive an empirical scaling for the width as a best fit to the data.

The width of the $\mathrm{H}$-mode edge steep gradient region was determined from Thomson scattering measurements of electron density and temperature and charge exchange recombination measurements of the ion temperature and $\mathrm{C}^{6+}$ density. Edge profile characteristics, such as the electron pressure gradient, were determined from these measurements by fitting to a hyperbolic tangent function including experimental uncertainties [10]. In general the width of the ion pressure steep gradient region is found to be equal to that of the electron pressure, and the more easily obtained electron pressure gradient scale length is used in the scaling studies. The $\mathrm{H}$-mode transport barrier is assumed to coincide with the high electron pressure gradient region. The scale length for $\mathrm{T}_{\mathrm{i}}$ is typically significantly larger than the pressure or density scale lengths. In addition to profile parameters, MHD equilibrium parameters, including local fields, were determined from external magnetic measurements using the EFIT code. The width scaling relations presented here apply to the interval between Type I ELMs; during Type I ELMs the width expands greatly.

In Shaing's model [11] the source of the $E_{R} \times B$ sheared is limited to the region of ion orbit loss across the plasma boundary and the transport barrier width is given by

$$
\delta_{\text {Shaing }}=\sqrt{\varepsilon / s} \rho_{\mathrm{p}}^{\mathrm{i}}
$$

where $\rho_{\mathrm{p}}^{\mathrm{i}}$ is the ion poloidal gyroradius and $\mathrm{s}$ is a term due to squeezing of the banana orbits by the radial electric field $s=\left|1-\frac{\mathrm{dE}_{\mathrm{R}}}{\mathrm{dR}} /\left(\Omega_{\mathrm{i}} \rho_{\mathrm{p}}^{\mathrm{i}}\right)\right|$. Near the top of the transport barrier where the width is limited $s \approx 1+\left(\rho_{\mathrm{p}}^{\mathrm{i}}\right)^{2} /\left(\mathrm{L}_{\mathrm{Ti}} \delta\right)$, assuming the bulk rotation terms are negligible in the radial force balance. Experimentally we observe $\rho_{\mathrm{p}}^{\mathrm{i}} / \delta=1$, however the ion temperature gradient scale length, $\mathrm{L}_{\mathrm{Ti}}$, is significantly larger than $\mathrm{d}$ so that orbit squeezing correction is small and $\delta_{\text {Shaing }} \approx \sqrt{\varepsilon} \rho_{\mathrm{P}}^{\mathrm{i}}=0.6 \rho_{\mathrm{P}}^{\mathrm{i}}$. Figure 1(a) shows a comparison of $\delta$ and $\rho_{\mathrm{P}}$, where $\rho_{\mathrm{P}}$ is determined 
assuming $\mathrm{T}_{\mathrm{e}}=\mathrm{T}_{\mathrm{i}}$ and deuterium. Fitting the data of Fig. 1(a) gives $\delta=(0.69 \pm 0.06) \rho_{\mathrm{P}}^{\mathrm{i}}$ in rough agreemwith Shaing's model, however fitting the data to a power law gives statistically improved fit and a weaker dependence on $\rho_{\mathrm{p}}^{\mathrm{i}}, \delta \propto\left(\rho_{\mathrm{p}}^{\mathrm{i}}\right)^{0.61 \pm 0.03}$. In particular there is very little dependence of $\delta$ on $\rho_{P i}$ in lower $q$ (lower $\left.\rho_{P}^{i}\right)$ discharges $\left(q=3.2=q_{\text {ITER }}\right)$.

In nonlinear gyrofluid simulations $[1,2,12]$ based on ITG and TEM mode it was found that turbulence was suppressed when the $\omega_{\operatorname{ExB}}>\gamma_{\mathrm{L}}$, where $\omega_{\operatorname{ExB}}$ is the velocity shear and $\gamma_{\mathrm{L}}$ is the linear growth rate for the ITG modes. Near the top of the pedestal $\omega_{\mathrm{E} \times \mathrm{B}} \approx \rho_{\mathrm{p}}^{\mathrm{i}} \mathrm{v}_{\text {thi }} /\left(\mathrm{L}_{\mathrm{Ti}} \delta\right)$, then using the expression given in [12] for $\gamma_{\mathrm{L}}$ gives

$$
\delta_{\text {IFS-PPPL }}=4 \rho_{\mathrm{i}}\left(\frac{\mathrm{T}_{\mathrm{i}}}{\mathrm{T}_{\mathrm{e}}}\right)\left(\frac{1+\max (0,(\mathrm{q}-3) / 15)}{1+\max (0, \varepsilon-0.17)}\right)\left(\frac{1}{1+\mathrm{L}_{\mathrm{Ti}} \mathrm{L}_{\mathrm{Ti}}^{\mathrm{CRIT}}}\right)
$$

where $\mathrm{L}_{\mathrm{Ti}}^{\mathrm{CRT}}$ is the critical ion temperature gradient scale length. Typically $\mathrm{L}_{\mathrm{Ti}}^{\mathrm{CRIT}} \approx \mathrm{a}$ [2], and $\mathrm{L}_{\mathrm{TT}}<<$ a, so we will neglect the critical gradient term. The functional dependence on $\mathrm{q}$ and $\varepsilon$ were determined for large aspect ratio, circular cross-section tokamaks and are not expected to be of exactly this form for ITER shape discharges; nevertheless, when this formula is applied with $q=$ q95 and $T_{i}=T_{e}$ it appears as an upper bound to the data as shown in Fig. 1(c). Again a weaker than linear dependence is found for a power law fit $\delta \propto \delta_{\text {IFS-PPPL }}{ }^{0.7}$, and there is an even weaker dependence for the lower q data.

Statistical analysis of the data set indicates that only $T_{e}^{P E D}, n_{e}^{P E D}$, and $I_{P}$, and related quantities are correlated with $\mathrm{d}$. In unpumped $\mathrm{H}$-mode discharges the average density and plasma current are proportional; since the density profile is flat in the core in $\mathrm{H}$-mode, $\mathrm{n}_{\mathrm{e}}^{\mathrm{PED}}$ is correlated with $\mathrm{I}_{\mathrm{P}}$ so that fits of $d$ cannot be made simultaneously to $\mathrm{T}_{\mathrm{e}}^{\mathrm{PED}}, \mathrm{n}_{\mathrm{e}}^{\mathrm{PED}}$, and $\mathrm{I}_{\mathrm{P}}$. A fit of $\mathrm{p}_{\mathrm{e}}^{\mathrm{PED}}$ and $\mathrm{B}_{\mathrm{P}}$ to $\delta$ which gives $\delta_{\mathrm{Pe}} \propto\left(\mathrm{P}_{\mathrm{e}}^{\mathrm{PED}}\right)^{0.52} /\left\langle\mathrm{B}_{\mathrm{POL}}\right\rangle^{0.94},\left(\left\langle\mathrm{~B}_{\mathrm{POL}}\right\rangle=\right.$ average over the separatrix) is of similar quality to fits of $\mathrm{d}$ to $\mathrm{T}_{\mathrm{e}}^{\mathrm{PED}}$ and $\mathrm{B}_{\mathrm{P}}$ giving $\delta \propto\left(\mathrm{T}_{\mathrm{e}}^{\mathrm{PED}}\right)^{0.36} /\left\langle\mathrm{B}_{\mathrm{POL}}\right\rangle^{0.44}$ where exponents are accurate to about \pm 0.1 . The fit of $\delta$ to $\mathrm{T}_{\mathrm{e}}^{\mathrm{PED}}$ is similar to the $\delta \propto \rho_{\mathrm{Pi}}^{0.6}$ fit shown in Fig. 1(a), while the fit to $\mathrm{P}_{\mathrm{e}}^{\mathrm{PED}}$ is equivalent the $\delta_{\mathrm{Pe}} \propto\left(\beta_{\mathrm{POL}}^{\mathrm{PED}}\right)^{0.5}$ or $\delta \propto \alpha_{\mathrm{CYL}}$ shown in Fig. 1(b), where $\alpha_{C Y L}=2 \mu_{0} R \frac{d p}{d R}\left(\frac{q_{C Y L}}{B_{T}}\right)^{2}\left(q_{C Y L}=\frac{a\left\langle B_{p}\right\rangle}{R B_{T}}\right)$ is the pressure gradient normalized as would be important for ballooning mode stability or magnetic well effects. 
(a)

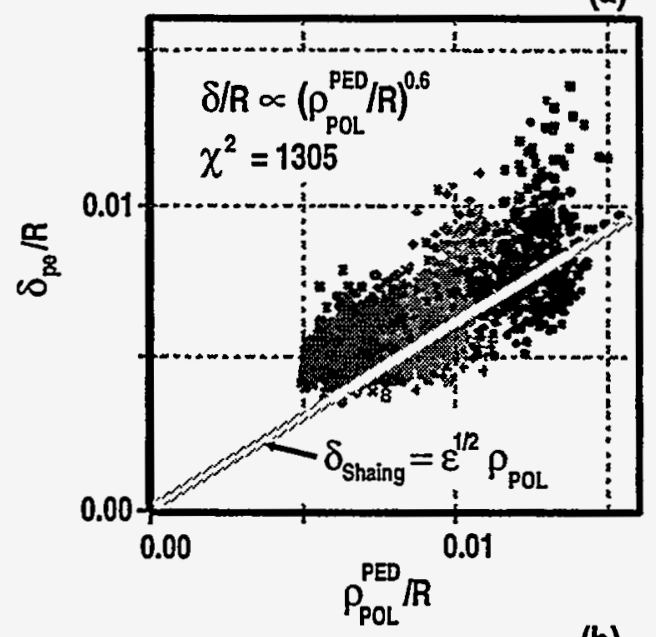

(b)

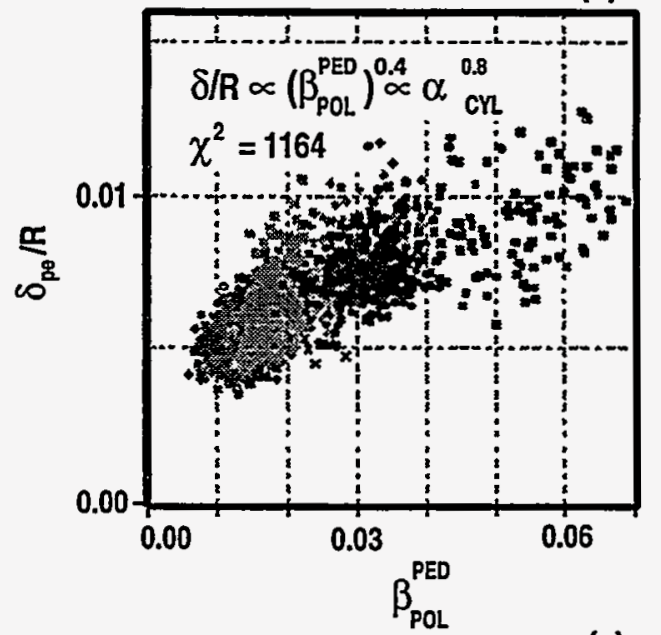

(c)

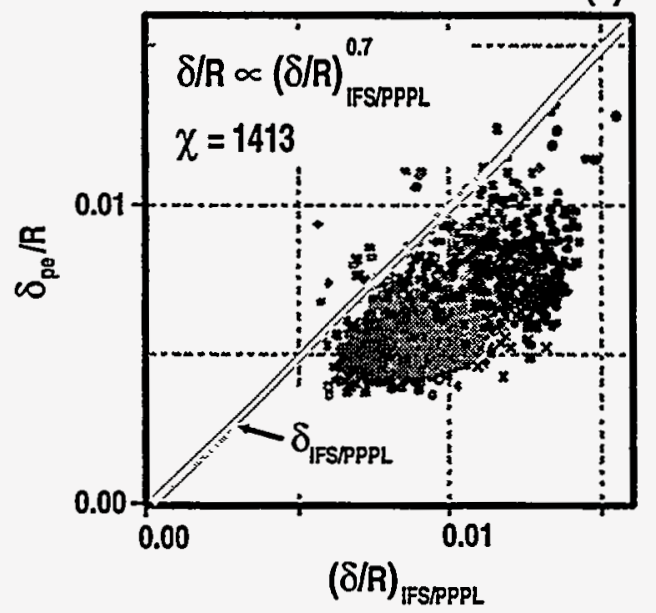

Fig. 1 . Width of the H-mode steep gradient region defined by the electron pressure on the outboard midplane between Type I ELMs: (a) versus poloidal gyroradius; the scaling derived by Shaing is in rough agreement with the data, (b) versus edge poloidal b, (c) versus the IFS/PPPL formula given in the text; this formula represents an upper bound to the data. Fits to power law of the three quanties are of similar quality. 
To determine whether a scaling of $d$ with $T^{\mathrm{PED}}$ or $\mathrm{p}^{\mathrm{PED}}$ was the more correct, we performed experiments in which divertor pumping was used to separate $\mathrm{n}^{\mathrm{PED}}$ and $\mathrm{I}_{\mathrm{p}}$ in $\mathrm{H}$-mode. In two discharges with otherwise similar parameters, Fig. $2, \delta_{\mathrm{pe}}$ remained fixed while $\mathrm{T}_{\mathrm{e}}^{\mathrm{PED}}$ varied a factor of two. In other discharges in which the density was pumped down continuously [10] we found that while a functional form of $\left(\mathrm{T}_{\mathrm{e}}^{\mathrm{PED}}\right)^{1.0}$ was required to fit the variation of $\mathrm{d}_{\mathrm{Pe}}$ between ELMs, a much weaker function $\left(\mathrm{T}_{\mathrm{e}}^{\mathrm{PED}}\right)^{0.2}$ was needed to match the variation of $\delta_{\mathrm{Pe}}$ throughout the shot, while $\left(\mathrm{P}_{\mathrm{e}}^{\mathrm{PED}}\right)^{0.5}$ fit both regimes. From these results we conclude that no function of $\mathrm{T}^{\mathrm{PED}}$ is likely to be consistent with the data.

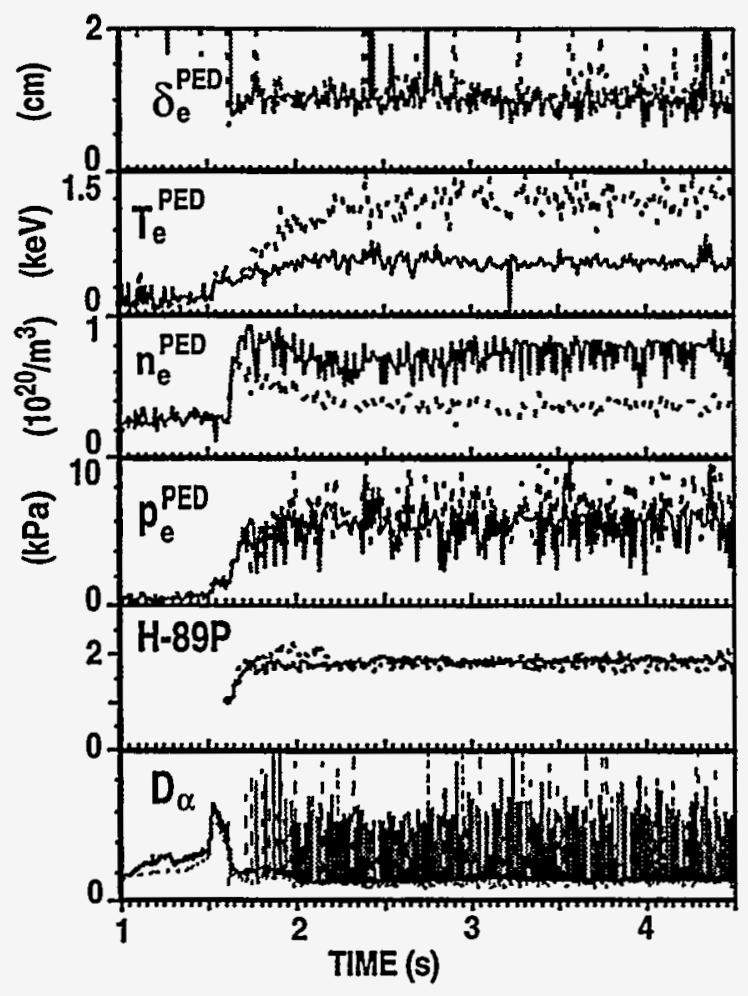

Fig. 2. Time evolution of the width of the $H$-mode steep gradient region defined by the electron pressure in the $H$-mode edge, $\delta_{e}^{P E D}$; the electron temperature, density and pressure at the top of the $H$-mode pedestal, $T_{e}^{P E D}$, $n_{e}{ }^{P E D}, p_{e}{ }^{P E D}$; the H-mode energy confinement enhancement factor, $H$, relative to the ITER 89P scaling; and, the $D_{\alpha}$ emission from the divertor region for a discharge without divertor pumping (solid line) and a discharge with divertor pumping (dotted line) showing that $\delta_{e}^{P E D}$ does not change with $T_{e}^{P E D}$. 


\section{SCALING OF THE EDGE PRESSURE GRADIENT IN H-MODE}

The pressure gradient that is reached before a type I ELM is generally thought to be given by the first stable limit for ideal infinite $n$ ballooning modes $[9,13]$. The stability of ballooning modes is usually described in terms of an S- $\alpha$ diagram [14] where $S$ is the magnetic shear and $\alpha$ is the normalized pressure gradient. In the first stable regime the marginal stability curve is given by $\alpha \propto S$, with the plasma is unstable at higher pressure gradients. Below a critical shear in noncircular cross-section tokamaks a region with no pressure gradient limit, referred to as the second stable regime, is encountered.

In the ITER shape discharges on DIII-D, we find that the electron pressure gradient normalized as for ballooning mode stability [14], $\alpha_{e}=2 \mathrm{P}_{\mathrm{e}}^{\prime} \mathrm{V}^{\prime}\left(\frac{\mathrm{V}}{2 \pi^{2} \mathrm{R}}\right)^{1 / 2} \frac{\mu_{0}}{4 \pi^{2}}$, (where $\left.{ }^{\prime}=\frac{\mathrm{d}}{\mathrm{d} \psi}\right)$ before a Type I ELM is relatively constant and independent of $q$, Fig. 3. This formula for $\alpha$ agrees with $\alpha_{C Y L}$ given in the previous section in the large aspect ratio, circular cross-section limit. For a fixed shape the magnetic shear profile, where $S=2 \frac{V}{V^{\prime}} \frac{q^{\prime}}{q}$, is relatively independent of $\mathrm{q}$ so that $\mathrm{S} / \mathrm{q}^{2}$ is mainly a function of $\mathrm{q}$. The roughly $30 \%$ variation in $\alpha$ at fixed $\mathrm{S} / \mathrm{q}^{2}$ represents a real ELM to ELM variation which typically increases with increasing q. Also shown in Fig. 3, is an approximate stability boundary for infinite $\mathbf{n}$ ideal ballooning modes computed with the BALOO code [15] based on equilibrium current density profiles determined using only magnetic measurements at the vacuum vessel wall. This stability calculation shows that high $\mathrm{q}$ discharges have access to the second stable regime for ideal ballooning as expected [16] below a critical value of $\mathrm{S} / \mathrm{q}^{2}$. However, access to second stability should allow higher $\alpha$ at high $\mathrm{q}$ and this is not observed. Furthermore, discharges at the low $q$ values ( $q=q$ ITER $=3.2$ ) are calculated in many cases to have electron pressure gradients which exceed the calculated total first stable limit; the ion pressure gradient in these cases is typically half the electron.

The seeming violation by the low $q$ discharges of the first stable limit for ideal infinite $n$ ballooning modes can be removed by using more realistic current density profiles. Theoretical studies [17] demonstrate that edge bootstrap current associated with the large edge pressure gradient can open second stable access in a local region near where the pressure gradient is maximum even in low $\mathrm{q}$ discharges with moderate shaping. This effect can be understood qualitatively from the fact that an additional toroidal current makes the shear more negative in the outboard bad curvature region by $\Delta S_{T} \approx-\mu_{0} a \Delta j_{T} / B_{p}$ while the effect of poloidal current is to make the shear more positive by $\Delta S_{p} \approx \mu_{0} a \Delta j_{p} / B_{T}$. For currents parallel to $\vec{B}$ the effect of the 


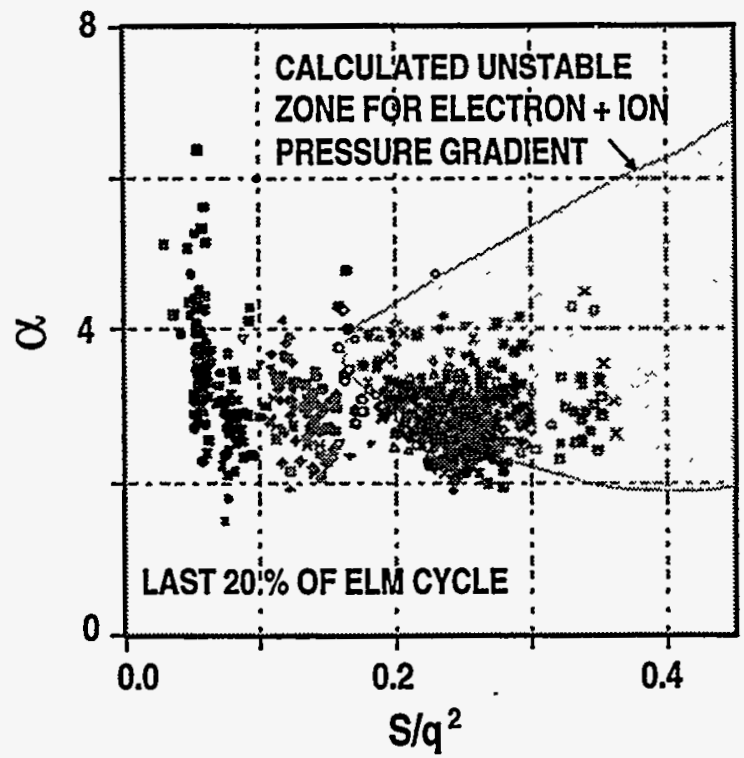

Fig. 3. Normalized edge pressure gradient, $\alpha$, just before a Type I ELM versus magnetic shear, $S$, over $q^{2}$. The experimental points include only the electron component while the stability boundary is for the total pressure gradient limit. The stability boundary is computed using a simpified current density profile which does not allow concentration of the edge current to the extent which can open access to second stability at high $S / q^{2}$. S is relatively fixed for this fixed shape so that the edge pressure gradient scales as would be expected for an ideal ballooning mode first stabile limit, however both the value of $\alpha$ and its behavior at low $S / q^{2}$ are not consistent with the calculated limit.

toroidal currents dominates by a factor of $\left(\mathrm{B}_{\mathrm{T}} / \mathrm{B}_{\mathrm{P}}\right)^{2}$. Similarly if the pressure gradient increases by $\Delta \alpha$ and this pressure increase were balanced by a ${ }_{\mathrm{T}} \times \mathrm{B}_{\mathrm{P}}$ force the change in $\mathrm{S}$ would be $\Delta S_{T} \approx-\Delta \alpha / \varepsilon$, while it would be $\Delta S_{p} \approx \Delta \alpha\left(B_{p} / B_{T}\right)^{2} / \varepsilon$ e if the same pressure change were balanced by a jp $\times B_{T}$. The bootstrap current is given approximately by $\mathrm{j}_{\| \mathrm{BS}} \approx \mathrm{C}_{\mathrm{BS}} \varepsilon^{1 / 2} \mathrm{R} \frac{\mathrm{dp}}{\mathrm{dR}}$, where $\mathrm{C}_{\mathrm{BS}}$ is a constant which may, for example, depend on collisionality. Thus as the pressure gradient grows its effect through the bootstrap current is to reduce the flux surface average shear by $\Delta \mathrm{S}_{\mathrm{BS}} \approx-\mathrm{C}_{\mathrm{BS}} \varepsilon^{-1 / 2} \Delta \alpha$, and if this shear change is large enough to reduce $\mathrm{S} / \mathrm{q}^{2}$ below the critical value for second stable access before a grows large enough to reach the first stable limit, then access to the second stable zone can be achieved.

To determine if the effect described above might be occurring in discharges with ITER shape and $\mathrm{q}$, MHD equilibria were generated using the EFIT code where, in addition to external magnetic measurements, the edge pressure profile profile was constrained to match the measured profile, and the edge current density was constrained to what would be expected from bootstrap plus Ohmic contributions. Although EFIT normally determines the current density profile by fitting to external magnetic measurements, it does not accurately separate the poloidal and toroidal current contributions to force balance on the short scale length of the edge pressure 
gradient. The pressure profile was input from the hyperbolic tangent fits including the ion contribution. The position of the pressure gradient relative to the separartix was set to be equal to the symmetry point of the hyperbolic tangent fit of the edge $T_{e}$ plus ' of its width. This location was found to agree with what would be expected from divertor constraints on open field lines from UEDGE modeling [18]. The edge toroidal current in the equilibrium was constrained to match the predictions from the ONETWO [19] transport code. Both a collisionless [20] and a collisional [21] form of the bootstrap current calculation was used. Equilibria were produced in this way for time shortly after the $\mathrm{L}$ to $\mathrm{H}$ transition and a time just before the first ELM. For both the collisional and collisionless bootstrap models access to the second stable region begins to open near the peak in the edge pressure gradient shortly after the L-H transition and the region with second stable access exists to accommodate the large pressure gradient at the time just before the ELM (Fig. 4). Using the ONETWO code we estimated the resistive skin time for the edge currents responsible for second stable access to be roughly $50 \mathrm{~ms}$ or less which is consistent with the time between ELMs in this case.

We also analyzed the equilibrium described above for low n ideal kink stability using the GATO code. In this case we find that the plasma is stable for the time slice just after the L-H transition but unstable to $\mathrm{n}=3$ and 4 while stable to lower $\mathrm{n}$ modes for the time slice just before the ELM. A model for a conducting wall at the position of the vacuum vessel was used in these calculations. The calculated mode was highly edge localized in the region of large pressure gradient and current density and has ballooning character (Fig. 5). In other work [22], we have found modes of this type to be destabilized by both increasing pressure gradient and current density. This is in contrast to high n peeling modes which are stabilized by increasing pressure gradient [23]. 
(a)
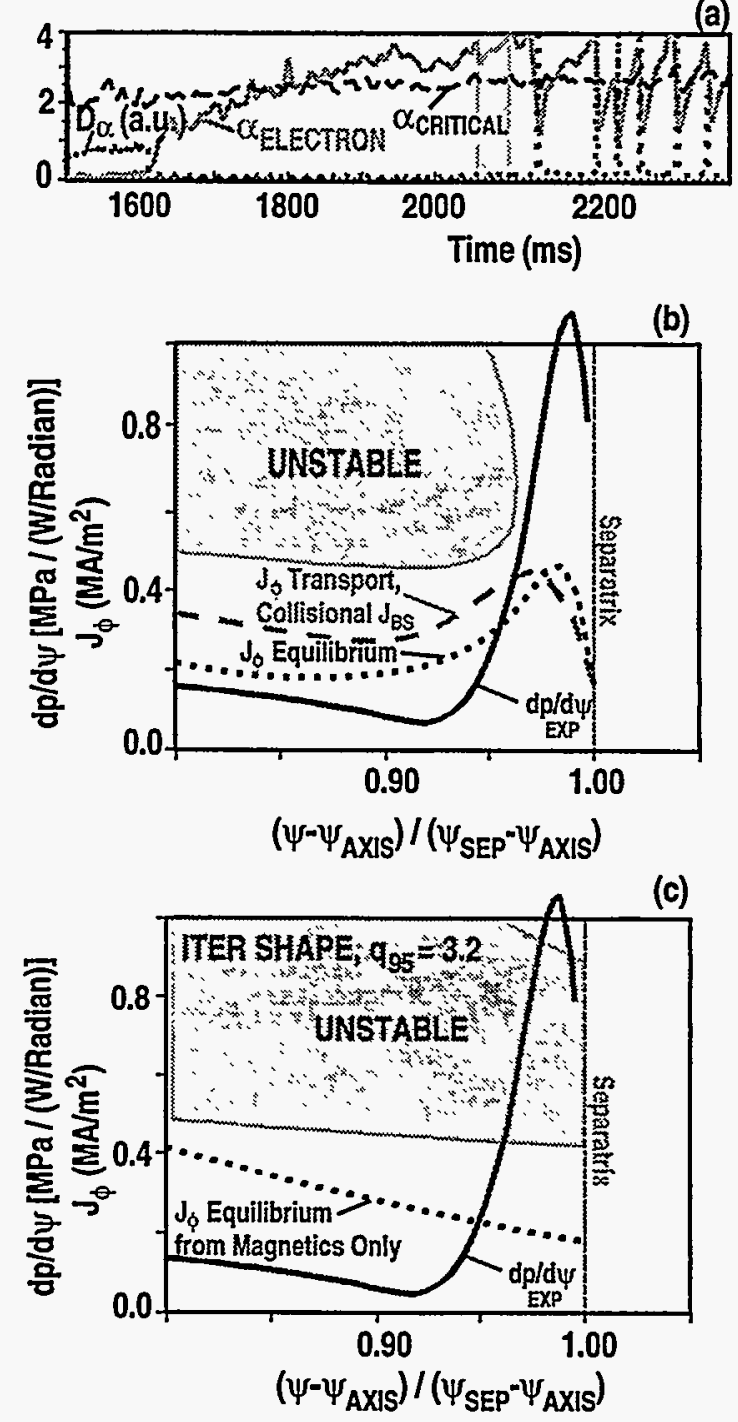

Fig. 4. Second stable access is achieved in low q discharges when a current density profile consistent with edge bootstrap current is used in the equilibrium fit. (a) Time history of the electron normalized pressure gradient $\alpha_{\text {ELECTRON }}$, the critical total pressure gradient computed for current density profiles determined from external magnetics only, $\alpha_{C R I T}$ and the $D_{\alpha}$ emmision from the divertor; $(b)$ edge pressure gradient is stable to ballooning modes due to opening of edge second stable access region caused by higher edge current (calculated at the time marked in (a), (c) edge pressure gradient would be unstable for flatter current density profiles such as those determined from external magnetics. 
(a)

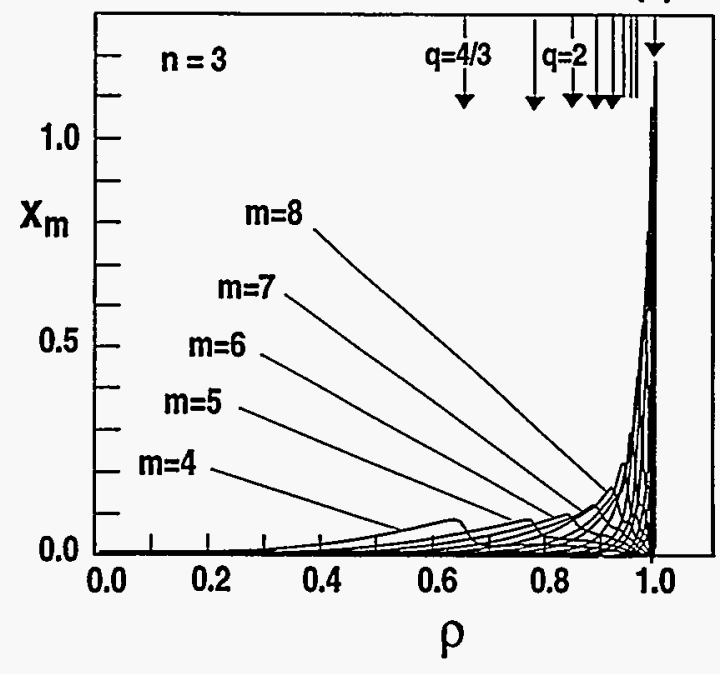

(b)

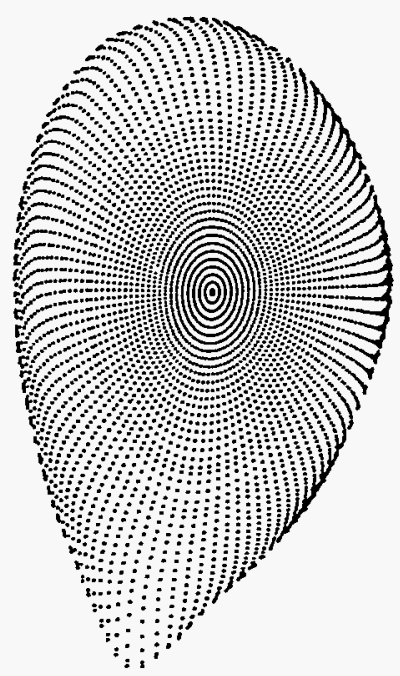

- Fig. 5. Edge localized $n=3$ ideal kink/ballooning mode determined to be unstable before the type I ELM at the time marked in Fig. 5. (a) Displacement $X_{M}=\xi \sqcup \nabla \psi$ for various m modes, and (b) two dimensional plot of displacement vectors $\xi$. 


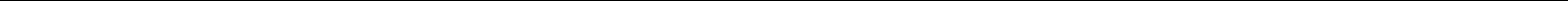




\section{DISCUSSION}

Although the data base analysis is consistent with a $\rho_{\mathrm{P}}$ scaling of the $\mathrm{H}$-mode transport barrier width, the divertor pumping experiments appear to rule out a simple scaling with edge temperature. A scaling of the form $\delta / \mathrm{R} \propto\left(\beta_{\mathrm{POL}}^{\mathrm{PED}}\right)^{1 / 2} \propto \alpha$ might occur due to a stabilizing effect of magnetic well on the instabilities involved in the turbulence [24]. Rogers and Drake [25] have shown however that nonlinear effects can result in the stabilization of some modes leading to an increase in the turbulent transport with $\alpha$. It is unlikely that $\delta / R$ is being set by the width of the region with second stable access at the edge which is also associated with the magnetic well. This width varies strongly with $q$, through the $S / q^{2}$ value required for second stable access, and such a strong $q$ dependence is not observed experimentally.

The large edge pressure gradients observed in the ITER shape discharges are consistent with the edge region having access to the second stable regime for ideal infinite $\mathrm{n}$ ballooning modes. Similar effects would be expected in ITER since the ballooning stability is related to the normalized quantities $S$ and $\alpha$. The effect of bootstrap current on the shear is related to the dimensionless pressure gradient, $\Delta S_{\mathrm{BS}} \approx-\mathrm{C}_{\mathrm{BS}} \varepsilon^{-1 / 2} \Delta \alpha$, and in fact may be larger in ITER since the collisionality in the edge of ITER should be less than about $20 \%$ of that in DIII-D.

The discharges are calculated to be unstable to low $n$ edge localized kink modes just before the ELM when more accurate pressure gradients and edge currents consistent with bootstrap current are used in the analysis. Precursor modes with $3<\mathrm{n}<15$ are commonly observed with magnetic probes for Type III ELMs on DIII-D and occasionally modes in this $\mathrm{n}$ range are observed before a type I ELM. Rapidly growing precursor modes with $1<\mathrm{n}<5$ are also observed before the VH-mode termination event [22]. The fact that precursor modes are generally not observed for type I ELMs may relate to the difficulty of detecting rapidly growing, slowly rotating, modes of somewhat higher $\mathbf{n}$. If these lower $\mathbf{n}$ kink modes are responsible for the ELM, their scaling to ITER is unclear, however it is typically found that the pressure gradient driven and current density driven $\beta$ limits scale similarly and thus one might expect a similar $\alpha$ value to limit the edge pressure gradient in ITER. Other ELM models such as peeling modes [23] or current diffusion ballooning mode turbulence 26] also have stability diagrams in S- $\alpha$ space and would be expected to result in the same $\alpha$ value for DIII-D and ITER. 


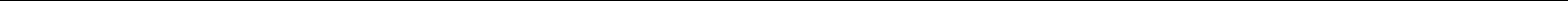




\section{REFERENCES}

[1] M. Kotschenreuter, W. Dorland, Q.P. Liu, G.W. Hammett, MA. Beer, S.A. Smith, A. Bondeson, S.C. Cowley, Proc. 16th International Conf. on Plasma Phys. and Controlled Nucl. Fusion Research, Montreal, Canada, October 1996, IAEA-F1-CN-64/D1-5 (to be published).

[2] R.E. Waltz, R.L. Dewar, X. Garbet, Phys. Plasmas 5 (1998) 1784.

[3] J.E. Kinsey, R.E. Waltz, and D.P. Schissel, Proc. 24th Euro. Conf. on Controlled Fusion and Plasma Physics, Physics (EPS, Berchtesgaden 1997) 21A Part III (1997) 1081.

[4] J.E. Kinsey and G. Bateman, Phys. Plasmas 3 (1996) 3344.

[5] S.I. Itoh, K. Itoh, A. Fukuyama, and M. Yagi, Phys. Rev. Lett. 72 (1994) 1200.

[6] T.H. Osborne, R.J. Groebner, L.L. Lao, A.W. Leonard, R. Maingi, R.L. Miller, G.D. Porter, D. Thomas, and R.E. Waltz, "H-mode Pedestal Characteristics, ELMs and Energy Confinement in ITER Shape Discharges on DIII-D," Plasma Phys. and Contr Fusion, (to be published 1998).

[7] A.W. Leonard, W. Suttrop, T.H. Osborne, T.E. Evans, D.N. Hill, A. Herrmann, C.J. Lasnier, D.N. Thomas, J.G. Watkins, W.P. West, M. Weinlich, H. Zohm, J. Nucl. Mat. 241-243 (1997) 628.

[8] K.H. Burrell, Phys. Plasmas 4 (1997) 1499.

[9] P. Gohil, M.Ali Mahdavi, L. Lao, K.H. Burrell, M.S. Chu, J.C. DeBoo, C.L. Hsieh, N. Ohyabu, R.T. Snider, R.D. Stambaugh, and R.E. Stockdale, Phys. Rev. Lett., 61 (1988) 1603.

[10] R.J. Groebner and T.H. Osborne, "Scaling Studies of the H-mode Pedestal," Phys. Plasmas (1998) (to be published).

[11] K.C. Shaing, Phys. Fluids B 4 (1992) 290.

[12] W. Dorland, M. Kotschenreuther, Q.P. Liu, M.A. Beer and G.W. Hammett, ISPP-17, Theory of Fusion Plasmas, J.W. Conner, E.Sindori, and J. Vaclavik (Eds.) SIF, Bologna 1996 p. 185.

[13] T. Kass, S. Gunter, M. Maraschek, W. Suttrop, H. Zohm, and the ASDEX Upgrade Team Proc. 24th Euro. Conf. on Controlled Fusion and Plasma Physics, Physics (EPS, Berchtesgaden 1997) 21A, Part IV, 1521 (1997). 
[14] J.W. Greene and M.S. Chance, Nucl. Fusion 21 (1981) 453.

[15] R.L. Miller, Y.R. Lin-Liu, A.D. Turnbull, V.S. Chan, L.D. Pearlstein, O. Sauter, and L. Villard, Phys. Plasmas 4 (1997) 1062.

[16] L.L. Lao, E.J. Strait, T.S. Taylor, M.S. Chu, T. Ozeki, W. Howl, R.D. Stambaugh, K.H. Burrell, M.S. Chance, J.C. DeBoo, P. Gohil, J.M. Green, R.J. Groebner, A.G. Kellman, M. Ali Mahdavi, T.H. Osborne, G. Porter, and A.D. Turnbull, Plasma Phys. Contr. Fusion 31 (1989) 509.

[17] R.L. Miller, Y.R. Lin-Liu, T.H. Osborne, and T.S. Taylor, "Ballooning Mode Stability for Self-Consistent Pressure and Current Profiles at the H-mode Edge", Plasma Phys. Control. Fusion (1998) (to be published)

[18] G.D. Porter, C.J. Lasnier, W. Meyer, J. Moller, "Characterization of the Edge Radial Profiles in H-mode in DIII-D," to be published 1998.

[19] W. Pfeiffer, F.B. Marcus, C.J. Armentrout, G.L. Jahns, T.W. Petrie, and R.E. Stockdale, Nucl. Fusion 25 (1985) 655.

[20] S.P. Hirshman, Phys. Fluids 31, 3150 (1988).

[21] W. A. Houlberg, private communication.

[22] J.R. Ferron, L.L. Lao, T.H. Osborne, D. Sauter, E.J. Strait, T.S. Talor, S.J. Thomson, A.D. Turnbull, Proc. of the 21th Europ. Conf. on Controlled Fusion and Plasma Physics (EPS, Montpellier 1994) 18B, Part I, 86 (1994).

[23] J.W. Conner, R.J. Hastie, H.R. Wilson, R.L. Miller, "Magnetohydrodnamic Stability of Tokamak Edge Plasmas," Phys. Plasmas (1998) (to be published).

[24] D.R. Dobrott and J.M. Greene, Plasma Phys. 17 (1975) 929.

[25] B.N. Rogers and J.F. Drake, Phys. Rev. Lett. 79 (1997) 229.

[26] S.-I. Itoh, K. Itoh, A. Fukuyama, and M. Yagi, Plasma Phys. Contr. Fusion 38 (1996) 527. 


\section{ACKNOWLEDGMENT}

Work supported by U.S. Department of Energy under Contracts DE-AC03-89ER51114, DEAC05-96OR22464 and W-7405-ENG-48. 